\title{
Expectativas profesionales de internos de medicina y su inclinación por la atención primaria de salud*
}

\author{
Professional expectations of Peruvian medical interns and their inclination for \\ primary health care
}

\author{
Marco Ramírez ${ }^{1}$ \\ ${ }^{1}$ Médico - Cirujano. Facultad de Medicina de San Fernando, Universidad Nacional Mayor de San Marcos (UNMSM). Lima, Perú. \\ * Trabajo presentado como Tesis para optar al Título Profesional de Médico Cirujano en la Facultad de Medicina -UNMSM
}

\begin{abstract}
Resumen
Introducción: Actualmente la disponibilidad de recursos humanos en salud se encuentra en los estándares internacionales, pero su distribución en nuestro país es inequitativa e inversa en relación a los problemas centrales de salud. Objetivo: Conocer las expectativas profesionales de los internos de medicina humana y su inclinación por la atención primaria de la salud. Diseño: Estudio descriptivo y transversal, con muestreo sistemático aleatorio. Lugar: Cinco hospitales nacionales de Lima y Callao, durante el año 2006. Participantes: Doscientos siete internos de medicina. Intervenciones: Aplicación de un cuestionario autoadministrado. Resultados: La especialización se mostró en $84,5 \%$ como la opción de formación y de ejercicio profesional más valorada y de prioridad para los internos de medicina. Entre las especialidades más anheladas se encontraron las quirúrgicas, en $37 \%$, y posteriormente las médicas, con $30 \%$. Asimismo, $30 \%$ de futuros médicos planeaba desempeñarse laboralmente en el extranjero. Se halló una asociación estadísticamente significativa entre la procedencia de una universidad pública y el deseo de realizar estudios de especialización en nuestro país, así como, la procedencia de una universidad privada y el deseo de realizar estudios de especialización en el extranjero. Finalmente, $25,1 \%$ de los internos manifiestó que sí se dedicarían a la atención primaria de la salud. Conclusiones: En los internos de medicina encuestados en el presente trabajo, existió una alta expectativa en la especialización, sobretodo quirúrgica, así como predisposición a laborar en el extranjero, con poca preferencia al trabajo en el primer nivel de atención.

Palabras clave: Especialización; atención primaria de salud; recursos humanos en salud.
\end{abstract}

\begin{abstract}
Introduction: Current availability of human resources in health is within international standards, but in our country distribution is not equitable and in inverse relation with central health problems. Objective: To determine professional expectations of medical interns and their inclination for primary health care. Design: Descriptive and transversal study, with randomized systematic sampling. Setting: Five Lima and Callao's national hospitals, during 2006. Participants: Two hundred and seven medical interns. Interventions: Application of a self-administered questionnaire. Results: Specialization represented in $84,5 \%$ the most valuable and priority training and professional practice option for medical interns. Most wished specialties were surgical in $37 \%$ and clinical in $30 \%$. Also $30 \%$ of future physicians planned to work in a foreign country. There was significant statistical association between procedence from a public university and the desire to specialize in our country, as well as, coming from a private university and wishes to specialize abroad. Finally $25,1 \%$ of the interns manifested they would do primary health care. Conclusions: There existed high expectative in doing a specialty in medical interns interviewed in this study, mainly in surgery, as well as predisposition to work in a foreign country, with little preference in first level health service.
\end{abstract}

Key words: Specialism; primary health care; health manpower.

\section{INTRODUCCIÓN}

La salud de la comunidad ha sido desde muchos años el objeto de la atención de los trabajadores de salud, cuyas acciones están dirigidas a satisfacer dichas necesidades de los individuos que la conforman y de esta forma ayudar a la comunidad a alcanzar un nivel de salud cada vez mejor. En la actualidad, el desarrollo de la tecnología ha llevado a los trabajadores de salud a proporcionar atención a problemas agudos y a grupos concentrados en las grandes ciudades, olvidándose de las zonas menos pobladas y asentamientos rurales ${ }^{(1)}$, lo que ha conducido a una organización médica centrada en el establecimiento hospitalario como modelo óptimo de servicio de salud ${ }^{(2,3)}$.

La educación médica, definida como la formación de profesionales en salud, no debe ser un proceso aislado, ya que se halla subordinada a la estructura económica predominante en la sociedad donde se desarrolla ${ }^{(4,5)}$. Es por ello que los programas de formación del médico general deben tener características propias en cada país y en cada región, en razón de las condiciones socioeconómicas locales ${ }^{(6)}$. Esto ha llevado a que los nuevos programas exijan la formación de personal de salud de todos los niveles y categorías, acorde con la problemática de salud de cada país ${ }^{(7)}$. En la actualidad, el estudiante de medicina dispone buen tiempo de su formación en las enseñanzas de médicos especialistas, quienes le trasmiten su particular ideología y, en algunas oportunidades, parcelada visión profesional. Es por ello que el futuro médico carece de una conceptualización y una experiencia totalizadora de la persona sana o enferma y una visión critica de su oficio, dentro de los cuales pueda luego insertar los eventuales desarrollos de la especialización ${ }^{(3,8)}$. Por otra parte, se está percibiendo que la principal motivación de los estudiantes de medicina para convertirse en médicos y elegir una determinada especialidad depende sobretodo de la gran demanda que existe en los estratos sociales altos por determinadas especialidades, que por una verdadera vocación, aspirando así a una mejor posición socioeconómica ${ }^{(9,10)}$.

Para una mejor atención en salud, los servicios sanitarios cuentan con niveles de atención, lo que optimiza sus resultados. Dentro de este esquema de atención se justifica la presencia del médico general con respecto al especialista y al hospital, con el médico general de filtro de la demanda que ha de consultar con el 
especialista ${ }^{(11,12)}$. El trabajo concatenado del médico general y del especialista facilita que tome contacto con el segundo nivel un grupo seleccionado de pacientes cuya prevalencia de enfermedad es mucho mayor que en el grupo sin seleccionar que toma contacto con el médico general. Con ello, se potencia el alto valor predictivo positivo que tiene el trabajo de los especialistas en su ámbito ${ }^{(13-15)}$.

En la Asamblea de la Organización Mundial de la Salud (OMS), en 1977, se definió y aceptó una política de salud denominada 'Salud para todos en el año 2000'. En 1978, en la reunión de Alma Ata, quedó establecido que para llevar a cabo dicha política era necesaria una estrategia denominada como atención primaria de salud (APS). Esta representa una estrategia para cubrir los principales problemas sanitarios de la comunidad, siendo el primer nivel de contacto de los individuos, la familia y la comunidad con el sistema nacional de salud, llevando lo más cerca posible la atención en salud al lugar de residencia y trabajo, promoviendo un autodesarrollo comunitario e individual al máximo posible y que implica, además del sector sanitario, a todos los sectores relacionados y a diferentes aspectos del desarrollo nacional y comunitario $^{(16)}$. Sin embargo, la atención primaria no ha afianzado una posición firme en el sistema de salud de muchos países, desde la pérdida de investigaciones sólidas en atención primaria hasta la falta de institucionalización en la educación médica. A pesar de muchas políticas de reforma, la atención primaria no ha llegado a cumplir con sus funciones cardinales y no logra consolidar una posición firme para el futuro ${ }^{(17)}$.

La fuerza de trabajo, elemento clave de todos los sistemas de salud, es fundamental para hacerla progresar. De manera global, todos los países, sean pobres o ricos, sufren de desajustes y desequilibrios numéricos, geográficos y de especialidades en su fuerza laboral de salud; desequilibrios que pueden resumirse como déficit global, mala distribución (agravada por la migración no planificada), ambientes inadecuados de trabajo, entre otros ${ }^{(18)}$. En la actualidad, la disponibilidad de recursos humanos se encuentra dentro de los estándares internacionales, pero su distribución es inequitativa e inversa en relación a los problemas centrales de salud. Reportes de la Dirección de Migraciones del Perú dan cuenta que los médicos migrantes pasaron de 4 416, en 1992, a 14 130, en el 2004; esto equivale a un aumento de $220 \%$. Por otra parte, Lima concentra el 53,19\% de médicos. Así mismo, se ha visto que la distribución de profesionales es inversa a los niveles de pobreza. Al no existir una política que regule el aumento de profesionales de salud y sus especialidades conforme a la demanda y necesidades, los incrementos producen resultados que no se compatibilizan con los requerimientos ${ }^{(19)}$. La precarización del empleo en salud con afectación del estatus de las carreras de salud, caracterizada por presupuesto sectorial bajo, salarios bajos y condiciones de trabajo precarias, son consecuencia del desequilibrio entre la oferta educativa y el mercado laboral; esto hace que aparezcan modalidades de contratación que vulneran los derechos humanos del trabajador y favorecen la migración de profesionales a otros países ${ }^{(20)}$.

Siendo los internos de medicina los futuros médicos de nuestro país, y la atención primaria uno de los ejes del mejoramiento de la salud en el Perú, es que nos propusimos conocer las expectativas profesionales de los internos de medicina y determinar su inclinación por la atención primaria de salud.

\section{MÉTODOS}

Se realizó un estudio observacional transversal. La población encuestada provino de cinco hospitales nacionales de Lima: Hospital Guillermo Almenara Irigoyen, Hospital Arzobispo Loayza, Hospital Daniel A. Carrión, Hospital Edgardo Rebagliati Martins y Hospital Dos de Mayo. Dichos hospitales fueron seleccionados debido a que cuentan con la mayor población de internos de medicina en nuestro país, los mismos que provienen de Lima y provincias, así como de diversas universidades de formación profesional. La población estuvo conformada por 392 internos de medicina que se encontraban realizando el internado médico durante el año 2006 y aceptaron participar en el estudio. Luego de realizar un muestreo sistemático de tipo aleatorio, se consiguió una muestra de 194 internos de medicina, los cuales se obtuvo de forma proporcionada a cada hospital (IC $=95 \%, \alpha=$ $0,05)$. Para mayor seguridad de obtener datos de la muestra necesaria indicada, se incrementó el número de participantes, quedando finalmente 207 internos de medicina humana, quienes dieron su consentimiento para participar del estudio y quienes llenaron debidamente el cuestionario.

Como instrumento de recolección de datos, se creó un cuestionario autoadministrado, con preguntas cerradas y abiertas, especialmente diseñado y cuyas principales variables a estudiar fueron: especialidades médicas de mayor prestigio, expectativas a futuro del ejercicio profesional, inclinación por la atención primaria de la salud y el tipo de universidad de procedencia. Dicho instrumento fue previamente validado mediante 'juicio de expertos', que se realizó con la participación de ocho colaboradores, médicos entendidos del tema de salud pública, gestión en salud y recursos humanos en salud, quienes revisaron el instrumento y su correlación con los objetivos que planteaba el estudio. Además, el cuestionario fue validado en una prueba piloto realizada en el Hospital Sergio Bernales. La aplicación del cuestionario se llevó a cabo el 11 de noviembre del 2006, en forma simultánea para los internos de cada hospital.

Con los datos obtenidos, se elaboró una base de datos, en el programa estadístico SPSS 11,1 para Windows, y se procedió a realizar análisis univariado (distribución de frecuencias, medidas de resumen) y análisis bivariado (chi-cuadrado o prueba exacta de Fisher).

\section{RESULTADOS}

Se encuestó 207 internos de medicina de 5 hospitales nacionales, cuya distribución por hospitales se puede apreciar en la Tabla 1. De la totalidad de la muestra, $96(46,4 \%)$ fueron del sexo femenino y $111(53,6 \%)$ del sexo masculino. La edad promedio de los internos encuestados fue 25 años, con un rango desde 22 a 36 años. Del total de encuestados, 135 (65,2\%) 
Tabla 1. Distribución de internos de medicina humana según hospital de procedencia.

\begin{tabular}{lcr}
\hline Hospital de procedencia & Frecuencia & \multicolumn{1}{c}{$(\%)$} \\
\hline Guillermo Almenara & 20 & 9,7 \\
Dos de Mayo & 59 & 28,5 \\
Arzobispo Loayza & 61 & 29,5 \\
Daniel A. Carrión & 33 & 15,9 \\
Edgardo Rebagliati & 34 & 16,4 \\
Total & 207 & 100,0 \\
\hline
\end{tabular}

internos procedían de universidades públicas y $72(34,8 \%)$, de universidades privadas. El 17,3\% procedía de universidades (públicas o privadas) de alguna provincia del país.

Acerca de su expectativa profesional, se realizó una pregunta de elección múltiple, donde indicaron -de manera no excluyente- las preferencias con respecto a lugares donde desearían trabajar. Del total de encuestados, 124 (59,9\%) internos desearían trabajar en un hospital general, $71(34,3 \%)$, en un instituto especializado, $57(27,5 \%)$ en una clínica privada, $23(11,1 \%)$, en un puesto o centro de salud, otros $23(11,1 \%)$, en un consultorio particular, y $3(1,4 \%)$, en otros lugares, como organismos no gubernamentales (ONGs), por ejemplo.

La distribución del área geográfica donde los internos deseaban realizar su ejercicio profesional se muestra en la Tabla 2.

Tabla 2. Expectativas sobre el lugar donde desearian realiza su ejercicio profesional los internos de medicina humana.

\begin{tabular}{lcr}
\hline Lugar de trabajo & Frecuencia & $(\%)$ \\
\hline La capital del país & 95 & 45,9 \\
Alguna provincia & 31 & 15,0 \\
Extranjero & 62 & 30,0 \\
Todavía no sabe & 19 & 9,2 \\
Total & 207 & 100,0 \\
\hline
\end{tabular}

En cuanto a los internos que desean trabajar en el extranjero, el país más anhelado fue España, luego le siguieron en orden descendente Estados Unidos, Brasil y Canadá.

Al investigar sobre cuáles son las actividades que desearían seguir dentro de su ejercicio profesional, los internos consideraron como prioridad seguir alguna especialidad, en $84,5 \%$ (175), quedarse como médico general, en 9,7\% (20), desea dedicarse a la investigación, en 3,4\% (7), introducirse en el campo de la salud pública, en 1,9\% (4), y dedicarse a la docencia, en $0,5 \%(1)$.

En cuanto el estudio de las especialidades más anheladas por los internos de medicina, se obtuvo que, entre las 39 especialidades futuras a elegir, las más preferidas fueron cirugía general, con $13,5 \%$ (28), pediatría, con $11,1 \%$ (23), gastroenterología, con $6,8 \%$ (14), ginecoobstetricia, con 6,3\% (13), cardiología, con 5,3\% (11), cirugía plástica, con 4,8\% (10), cirugía cardiovascular, con $4,8 \%$ (10), y otras especialidades, 47,4\% (98). La distribución de las especialidades anheladas por los internos según las grandes áreas de la medicina se muestra en la Figura 1.

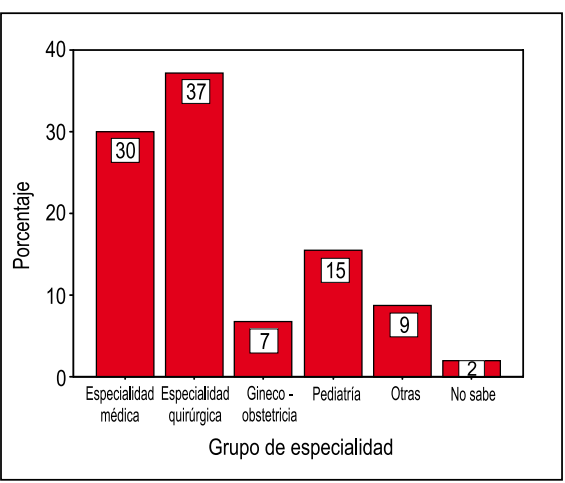

Figura 1. Distribución porcentual de las especialidades más anheladas por los internos de medicina, agrupadas según áreas de la medicina.

Asimismo, acerca del lugar donde pensaron realizar los internos de medicina sus estudios de especialización, se obtuvo los datos consignados en la Tabla 3.

En cuanto a los que desearían realizar su especialización en el extranjero, la mayoría tiene como principales opciones realizarla en España, Estados Unidos y Brasil.

Entre las especialidades médicas que los internos consideran que tienen mayor prestigio socioeconómico, se encuentran

Tabla 3. Lugares donde han pensado realizar sus estudios de especialización los internos de medicina.

\begin{tabular}{lcr}
\hline Lugar de especialización & Frecuencia & $(\%)$ \\
\hline Dentro del país & 96 & 46,4 \\
En el extranjero & 77 & 37,2 \\
Todavía no sabe & 34 & 16,4 \\
Total & 207 & 100,0 \\
\hline
\end{tabular}

las del área de especialidades quirúrgicas. Según la pregunta de opción múltiple -no excluyente- sobre qué especialidad quirúrgica considera la de mayor prestigio, como primera especialidad se encuentra la cirugía plástica, con 46,8\% (97), seguida de la cirugía de tórax y cardiovascular, con $41,5 \%$ (86), y la neurocirugía, con $24,7 \%(72)$.

Sobre la atención primaria de la salud, 131 internos contestaron que sí conocen el concepto de atención primaria de la salud (APS). De estos, 78,6\% (103) poseía un conocimiento inadecuado, $16,8 \%$ (22) poseía un conocimiento adecuado y 4,6\% (6) dejó la pregunta en blanco.

Al preguntar a los 131 internos sobre la importancia que le asignan a la APS en la formación y práctica médica en nuestro país, se obtuvo que $64,8 \%$ (85) piensa que es muy importante, entre otras cosas "porque de esa manera se daría un adecuada atención básica y disminuiría la afluencia a hospitales generales", "porque es necesario en un país subdesarrollado como el nuestro con pocos recursos", "porque desde un nivel primario se pueden evitar muchas patologías y mejoraría la política de salud". El resto de participantes lo considera de importancia media o no le concede importancia.

De los 131 internos, 52 (39,7\%) refirieron que si se dedicarían a la APS, esto representa realmente el 25,1\% del total de internos encuestados. Entre los principales motivos que manifiestan los internos por los cuales no se dedicarían a la APS refieren: "Debo realizar alguna especialización", "Muy mala remuneración económica", "No es adecuadamente valorada ni recompensada en este país", "Hay mucho déficit", "No es rentable, no hay mucho apoyo de parte del ministerio al médico en este sector".

$\mathrm{Al}$ asociar los grupos de especialidades que desearían seguir los internos de medicina según el tipo de universidad de procedencia, se obtuvo que $34 \%$ (46) de los 135 internos de universidades públicas desearía seguir alguna especialidad médica y 31,8\% (43) alguna especialidad quirúrgica, mientras que del total de los 72 internos de universidades privadas, la mayoría desea seguir alguna especialidad quirúrgica, $47,2 \%(34)(p=0,276)$. Figura 2. 


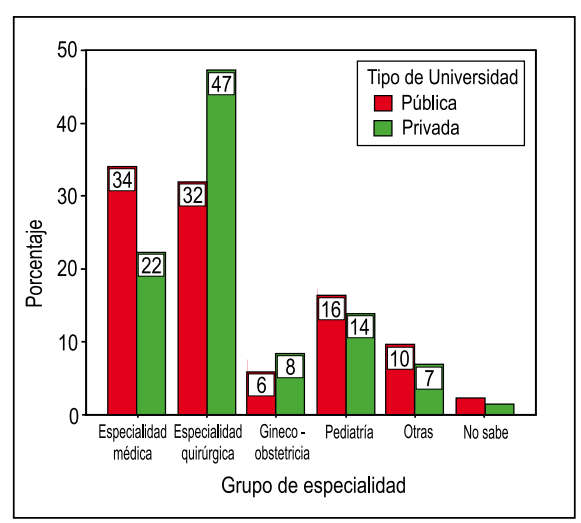

Figura 2. Distribución porcentual por área de las especialidades más anheladas según tipo de universidad de procedencia.

Al analizar los resultados obtenidos con respecto al lugar donde los internos desearían realizar su especialización, se encontró un asociación significativa ( $p=$ 0,002 ) entre estas variables, ya que el mayor porcentaje de internos de universidades públicas $(54,8 \%)$ deseaba realizar sus estudios de especialización dentro del país, mientras que la mayoría de internos de universidades privadas $(51,3 \%)$ lo deseaba realizar en el extranjero. Figura 3.

Al relacionar las especialidades más anheladas según genero del interno de medicina, se encontró que las más anheladas por el sexo femenino fueron pediatría, con $15,6 \%$ (15), cirugía general, con $11,4 \%$ (11), y ginecoobstetricia, con 8,3\% (8). Mientras que, las más anheladas por el sexo masculino fueron cirugía general, con 15,3\% (17), y cirugía cardiovascular, gastroenterología y pediatría, con 7,2\% (8) $(p=0,164)$.

Al correlacionar la especialidad que consideran de mayor prestigio, los internos de medicina y el grupo de especialidad al cual desearían dedicarse, se obtuvo que $27,8 \%$ (56) de los internos consideraba de mayor prestigio socioeconómico y a su vez deseaba dedicarse al área de las especialidades quirúrgicas. Así mismo, se observó que, si bien los internos deseaban seguir otras especialidades, estos consideraban de mayor prestigio al grupo de las especialidades quirúrgicas.

Al relacionar la inclinación de los internos de medicina según el tipo de conocimiento que tienen sobre la atención primaria de la salud, se observó que

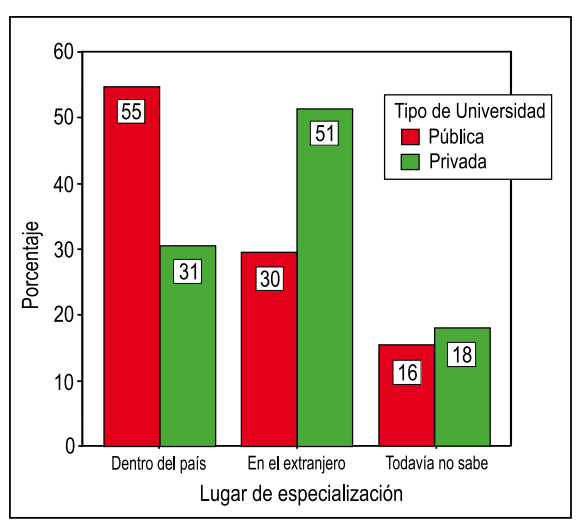

Figura 3. Distribución porcentual del lugar anhelado de especialización según tipo de universidad de procedencia.

$15,3 \%$ (8) de los que refirieron que se inclinarían por la APS tiene un conocimiento adecuado, mientras que $84,6 \%$ (44) posee un conocimiento inadecuado o no contestó.

\section{DISCUSIÓN}

En el presente estudio se encontró que la gran mayoría de los internos de medicina desea desempeñar su labor asistencial en el tercer o cuarto nivel de atención y solo un reducido $11,1 \%$ muestra alguna inclinación por el primer nivel de atención. Asimismo, se puede observar que un importante $30 \%$ de futuros médicos tiene pensado aplicar sus conocimientos -formados en las escuelas de medicina del Perú- en el extranjero. Esto va muy de la mano con lo que se puede apreciar actualmente con respecto al fenómeno de migración de los profesionales de la salud. Reportes de la Dirección de Migraciones dan cuenta que los médicos migrantes pasaron de 4416 en 1992 a 14130 en el 2004, esto es un aumento de 220\% ${ }^{(19)}$. España y Estados Unidos son los principales países que tienen en mente los internos de medicina para ejercer su profesión; esto se correlaciona con los principales destinos donde han migrado los profesionales médicos desde hace 10 años: EEUU 33,2\%, Chile 12,2\%, Bolivia 11,7\%, Ecuador 7,5\%, Argentina 7,7\%, Brasil 6,7\% y España $3,9 \%{ }^{(21)}$. Este deseo de emigrar obedece a una realidad a nivel mundial donde el personal de salud proveniente de países de recursos bajo y mediano buscan en los países desarrollados, como Estados
Unidos, Inglaterra, Canadá, entre otros, mejores oportunidades y una mejor situación socioeconómica ${ }^{(22,23)}$. Por otra parte, $45,9 \%$ de los internos de medicina deseaba laborar en la capital del país y solo $15 \%$ había pensado en alguna provincia como posibilidad, entre ellas Trujillo, Arequipa y Ancash. Como se puede apreciar, persiste el afán de seguir concentrando mayor número de médicos en la capital, dejando descuidada nuestras provincias, donde más atención en salud se necesita. Esta desigualdad en la distribución de recursos humanos en salud ha hecho que actualmente haya un total de 19 departamentos (79\%) con déficit de médicos y solo 5 departamentos (21\%) del país con una razón superior a 10 por 10000 habitantes, concentrando Lima el 53,19\% de médicos ${ }^{(19,24)}$.

Con respecto al tema de la especialización, esta se mostró en un $84,5 \%$ como la opción de formación y de ejercicio profesional más valorada y de mayor importancia para los internos de medicina y como segunda opción, con $31,4 \%$, colocaban a la medicina general. Entre las especialidades, las más anheladas eran las quirúrgicas, en 37\%, y posteriormente las médicas, con $30 \%$. Este gran porcentaje de alumnos que desea seguir una especialidad se refleja en varios estudios en diferentes partes del mundo. Así, en un estudio suizo realizado en 3 universidades, $56,6 \%$ ya había decidido seguir alguna especialidad ${ }^{(25)}$. En un estudio inglés realizado en médicos jóvenes graduados en 1993, al año y a los 3 años de culminar sus estudios, mostró que $68,9 \%$ deseaba continuar alguna especialidad (21,5\% médicas y $16,9 \%$ quirúrgicas) y $25,8 \%$ deseaba la medicina general. Estos datos variaron un poco a los 3 años, cuando $66 \%$ deseaba una especialidad (18,4\% médicas y $13,6 \%$ quirúrgicas) y, el porcentaje de los que deseaban medicina general, se incrementó a $29,1 \%{ }^{(2)}$. En otro estudio inglés realizado en los graduados de las escuelas de medicina, los años 1996 y 1999, reveló que $76 \%$ deseaba alguna especialidad médica, mientras que $23 \%$ deseaba continuar como médico general (8). Otro estudio más reciente, realizado en una población de 2778 médicos graduados el 2002, en Inglaterra, que busca- 
ba determinar cuáles eran las elecciones de especialidad médicas, encontró que $22,7 \%$ ( $28,1 \%$ de hombres y $14,5 \%$ de mujeres) deseaba continuar como médico general, $31,1 \%$ de los hombres y un $11,9 \%$ de las mujeres prefería una especialidad quirúrgica, $0,7 \%$ de hombres y $3,4 \%$ de mujeres elegía ginecoobstetricia $y$, finalmente, $3,4 \%$ de hombres y $7,9 \%$ de mujeres optaba por pediatría (26). Finalmente, un estudio encontró que la elección por la medicina general había incrementado ( $14,2 \%$ a $21,8 \%)$, mientras que la elección por las especialidades quirúrgicas había disminuido $(22,2 \%$ a $10 \%$ ); no hallaron cambios significativos en las especialidades pediátricas, médicas y ginecoobstetricia ${ }^{(27)}$.

En América Latina se han realizado pocos estudios al respecto. En los años 60, el médico argentino Juan César García analizó el proceso de especialización en 6 escuelas de medicina de América Latina, encontrando que al inicio de los estudios de medicina ya $52 \%$ de los estudiantes tenía en mente una determinada especialidad, mientras que al final de sus estudios esta decisión llegó al 66\%. Un estudio más reciente realizado en Argentina, en el año 2001, halló que los jóvenes que se decidieron por medicina expresaban su intención por especializarse desde su ingreso a la carrera (74\%) hasta el fin de ella (100\%), siendo las especialidades quirúrgicas las más anheladas ${ }^{(28)}$. La información obtenida en el presente estudio obedece a la realidad de cómo están distribuidos los recursos humanos en salud por especialidad. Los médicos especialistas presentaron un aumento en los últimos 15 años; para el año 2004, el Colegio Médico del Perú registró 19479 especialistas. La mayor cantidad de especialistas registrados corresponde a las denominadas especialidades básicas: ginecoobstetricia (2 008), pediatría (1 841), cirugía general (1 429), medicina interna (1 349), anestesiología (874) y oftalmología (666) ${ }^{(29)}$.

De todos los internos de medicina que participaron en el estudio, 131(63,2\%) contestaron que sí conocen en qué consiste la estrategia de la atención primaria de la salud; sin embargo, de ellos solo $16,8 \%$ (22) poseía un conocimiento adecuado al respecto. El 15,3\% (8) de los que refirieron que se inclinarían por la APS tenía un conocimiento adecuado, mientras que $84,6 \%$ (44) poseía un conocimiento inadecuado o no contestó. Estos datos son similares con los hallados por un estudio argentino, donde la mayoría de los ingresantes a las escuelas de Medicina ignoraba el significado de la APS y solo $0,5 \%$ de los estudiantes del último año presentaba alguna inclinación hacia ella ${ }^{(28)}$. El poco conocimiento y la poca motivación por la atención primaria de la salud obedece a los grandes problemas y desafíos que desde sus inicios tuvo la APS. En un documento de la Organización Panamericana de la Salud sobre renovación de la atención primaria de la salud en las Américas, refiere que los desafíos actuales son: los trabajadores de salud calificados son insuficientes para proveer cobertura universal, los profesionales calificados prefieren trabajar en hospitales y ciudades, carencia de apoyo y supervisión adecuada, no hay desarrollo ni estímulos suficientes, el entrenamiento en pregrado y posgrado del personal de salud no es coherente con los requerimientos de la práctica de la APS, dificultades inherentes a la transformación del sector salud, desde los enfoques curativos, basados en los hospitales, a los preventivos, basados en las comunidades ${ }^{(30)}$.

Los resultados obtenidos con respecto al proceso de elección de una especialidad médica corroboran lo encontrado en otros estudios; elegir una especialidad médica o quirúrgica es un proceso con muchas preguntas, decisiones, expectativas y necesidades en diferentes partes del mundo y está relacionado al contexto sociocultural y educativo de los estudiantes de medicina. Los hallazgos indican que la transición desde las escuelas de medicina hasta la residencia, no solo es el género o las habilidades profesionales, sino también la motivación interior y exterior, lo que contribuye al éxito académico y profesional.

Un dato interesante que se encontró en el presente trabajo fue la diferencia significativa en cuanto al lugar donde realizar su especialización. Mientras que los estudiantes provenientes de universidades particulares deseaban realizarla en el extranjero, la mayoría de los internos de universidades públicas deseaba realizarla en el país. Esto obedece, además de las posibilidades económicas de cada estudiante, a la realidad que se vive actualmente en el país, desarticulación entre el mundo del trabajo y el de la formación en salud, lo que da origen a una sobreoferta de egresados. Con esto, se observa el subempleo y desempleo en salud y la búsqueda de nuevas soluciones, entre ellas la migración de profesionales en salud, algunas veces solo para realizar la especialización y muchas otras para finalmente quedarse a ejercer y formar parte de la fuerza de trabajo en salud de otros países. La precarización del empleo, como consecuencia de la sobreoferta educativa en el país y el desequilibrio entre la oferta de profesionales de salud y el mercado laboral, favorecería las causas de la migración hacia países con mejores condiciones de vida y desarrollo.

Concluimos, del presente trabajo, que existe una alta expectativa en la especialización en los internos de medicina, sobre todo quirúrgica, así como predisposición a laborar en el extranjero, con una baja preferencia al trabajo en el primer nivel de atención. Asimismo, consideramos necesario potenciar la educación en el tema del primer nivel de atención, para de ese modo inculcar en los futuros médicos de nuestro país el desarrollo laboral en ese nivel, con la consiguiente mejora en nuestros servicios de salud y una equitativa distribución de recursos humanos.

\section{AGRADECIMIENTOS}

Al Dr. José Miguel Arca González del Valle, docente del Departamento de Medicina Preventiva y Salud Pública de la Facultad de Medicina UNMSM, por ser asesor del presente trabajo. Y a la Unidad de Investigación de la UNMSM, por el financiamiento del proyecto en el año 2006.

\section{REFERENCIAS BIBLIOGRÁFICAS}

1. Peña FM, Puente A. Formación del personal de enfermería para la atención de salud a la comunidad. Educación Médica y Salud. 1980;14(1):41-54.

2. Goldacre M, Lambert T. Stability and change in career choice of junior doctor: postal questionnaire of the Unites kingdom qualifiers of 1993. Medical Education. 2000;34:700-7. 
3. Neri A. La medicina en el mundo contemporáneo. Educación Médica y Salud. 1979;13(2):113-33.

4. García JC. La Educación Médica en América Latina. Capitulo I: Análisis de la Educación Médica en América Latina. Washington, DC: Organización Panamericana de la Salud; 1972. p. 1-14.

5. Salaverry 0. Una visión histórica de la educación médica. An Fac med [revista en Internet]. 1998;59(3). Disponible en: http://sisbib.unmsm. edu.pe/BVRevistas/anales/v59_n3/edu_medica. htm

6. Fraga C, Rosa A. Formacao e treinamiento do medico general. Educación medica y salud. 1979;13(2):149-63.

7. Stern D, Papadakis M. The developing physician -becoming a professional. N Engl J Med. 2006;355:1794-9.

8. Lambert T, Davidson J, Evans J, Goldacre M. Doctors'reasons for rejecting initial choice of specialities as long-term careers. Medical Education. 2003;37:312-8.

9. Vaglun P, Wiers-Jennsen J, Ekeberg 0. Motivation for medical school: the relationship to gender and speciality preferences in a nationwide sample. Medical Education. 1999;33:236-42.

10. Kassebaum D, Szenas P. Factors influencing the specialty choices of 1993 medical school graduates. Academic Medicine. 1994;69(2):16370.

11. Ortún V, Gérvas J. Fundamentos y eficiencia de la atención médica primaria. Med Clín (Barc). 1996;106:97-102.

12. Knottnerus JA. Medical decision making by general practitioners and specialists. Fam Pract. 1991;8:305-7.

13. Edwards MW, Forman WM, Walton J. Audit of abdominal pain in general practice. J R Coll Gen Pract. 1985;35:235-8.

14. Sox HC, Hickam DH, Marton KI, Moses L, Skeff $\mathrm{KM}$, et al. Using the patient's history to estimate the probability of coronary artery disease: a comparison of primary care and referral practices. Am J Med, 1990;89;7-14.

15. Gérvas J, Pérez M. El fundamento científico de la función de filtro del médico general. Rev Brasilera Epidemiol. 2005;8(2):205-18.
16. Kroeger A, Luna R. Atención Primaria de Salud. Capitulo I: Aspectos programáticas de la atención primaria de salud a nivel nacional e internacional. Washington, DC: Organización Panamericana de la Salud; 1992. p. 5-20.

17. Starfield B, Oliver T. Primary care in the United States and its precarious future. Health and Social Care in the Community. 1999;7(5):315-23.

18. Ministerio de Salud, Instituto de Desarrollo de Recursos Humanos. Situación de los Recursos Humanos y Plan de una Década para su Desarrollo (Documento de Síntesis). Capitulo II: La Fuerza Laboral y su Rol Central en Salud. Lima: Minsa; 2006. p. 7-8.

19. Ministerio de Salud, Instituto de Desarrollo de Recursos Humanos. Situación de los Recursos Humanos y Plan de una Década para su Desarrollo (Documento de Síntesis). Capitulo V: El Estado de Situación: Agenda de problemas en el Perú. Lima: Minsa; 2006. p. 10-9.

20. Ministerio de Salud, Instituto de Desarrollo de Recursos Humanos. El Perú respondiendo a los desafíos de la Década de los Recursos Humanos en Salud. Capitulo I: Antecedentes. Lima: Minsa; 2006. p. 13-9.

21. Ministerio de Salud, Instituto de Desarrollo de Recursos Humanos. El Perú respondiendo a los desafíos de la Década de los Recursos Humanos en Salud. Capítulo IV: Evaluación del Movimiento migratorio de Profesionales en salud, Perú. 1994-2005. Lima: Minsa; 2006. p. 59-75.

22. Pond B, McPake B. The health migration crisis: the role of four organisation for economic cooperation and development countries. Lancet. 2006;367:1448-55.

23. Fitzhugh M. The metrics of the physician brain drain. NEJM. 2005;353:1810-8.

24. Ministerio de Salud, Instituto de Desarrollo de Recursos Humanos. El Perú respondiendo a los desafíos de la Década de los Recursos Humanos en Salud. Capitulo II: Distribución de los Recursos humanos, un desafío para la equidad en Salud en el Perú. Lima: Minsa; 2006. p. 21-40.

25. Buddeberg-Fischera B, Klaghofera R, Abelb T, Buddeberga $C$. The influence of gender and personality traits on the career planning of Swiss medical students. Swiss Med Wkly. 2003;133:53540.

26. Lambert T, Goldacre M, Turner G. Career choices of United Kingdom medical graduates of 2002: questionnaire survey. Medical Education. 2006;40(6):514-20.

27. Lambert T, Goldacre M, Turner G. Career choices of United Kingdom medical graduates of 1999 and 2000: questionnaire surveys. BMJ. 2003;326:1935.

28. Carrera L, Enría G, D’Ottavio A. La atención primaria de la salud y la especialización médica: ¿Categorías opuestas o complementarias?. Educación Médica. 2004;7(4):132-9.

29. Ministerio de Salud, Instituto de Desarrollo de Recursos Humanos. Situación y Desafíos de los Recursos Humanos en Salud. Gobernabilidad y desempeño con desarrollo humano. Capitulo 2: Información Básica sobre Recursos Humanos en salud. Lima: Minsa; 2005. p. 43-72.

30. Organización Panamericana de la Salud. Renovación de la Atención Primaria de la Salud en las Américas. Documento de posición de la OPS/ OMS. Capitulo III: El Camino a seguir. Washington DC: OPS/OMS; 2005. p.17-23. Disponible en: http://www.ops-oms.org/spanish/ad/ths/os/APSdocumentoposicion19-07-05.pdf

Manuscrito recibido el 25 de junio de 2008 y aceptado para publicación el 20 de agosto de 2008.

Correspondencia:

Marco Ramírez Huaranga

Jirón Morona 538. Urb. Chacra Colorada

Lima 5, Perú

Correo-e: hurauma@hotmail.com 